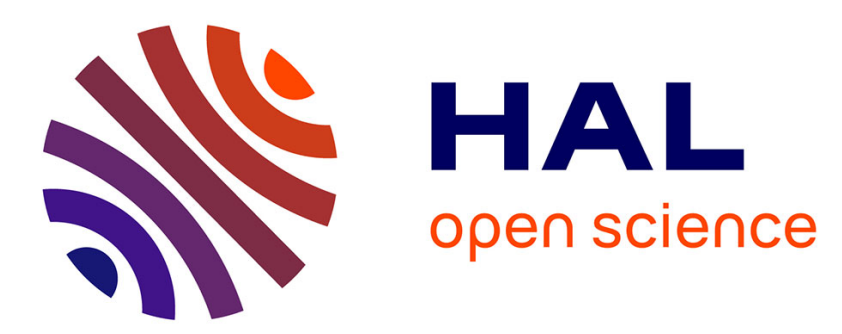

\title{
Brain-Computer Interface as Networking Entity in Body Area Networks
}

\author{
M. A. Lopez-Gordo, F. Pelayo Valle
}

\section{To cite this version:}

M. A. Lopez-Gordo, F. Pelayo Valle. Brain-Computer Interface as Networking Entity in Body Area Networks. 13th International Conference on Wired/Wireless Internet Communication (WWIC), May 2015, Malaga, Spain. pp.274-285, 10.1007/978-3-319-22572-2_20 . hal-01728816

\section{HAL Id: hal-01728816 https://hal.inria.fr/hal-01728816}

Submitted on 12 Mar 2018

HAL is a multi-disciplinary open access archive for the deposit and dissemination of scientific research documents, whether they are published or not. The documents may come from teaching and research institutions in France or abroad, or from public or private research centers.
L'archive ouverte pluridisciplinaire HAL, est destinée au dépôt et à la diffusion de documents scientifiques de niveau recherche, publiés ou non, émanant des établissements d'enseignement et de recherche français ou étrangers, des laboratoires publics ou privés. 


\title{
Brain-computer Interface as networking entity in Body Area Networks
}

\author{
M. A. Lopez-Gordo ${ }^{1,2,3}$, F. Pelayo Valle ${ }^{4}$ \\ ${ }^{1}$ Dept. of Engineering in Automatic and Electronics, Electronic, Architecture of Computers \\ and Networks, University of Cadiz, (Spain) \\ ${ }^{2}$ Dept. of Signal Theory, Communications and Networking, \\ University of Granada, 18071, (Spain) \\ ${ }^{3}$ Nicolo Association, Churriana de la Vega, Granada (Spain) \\ malg@nicolo.es \\ ${ }^{4}$ Dept. of Computer Architecture and Technology, University of Granada, \\ c/ Periodista Daniel Saucedo, 18071, Granada, Spain. \\ fpelayodugr.es
}

\begin{abstract}
.
Modern BCIs are non-invasive, wireless, wearable and EEG-based systems capable to transduce brain signals into cognitive information and stream it out in form of data. BCIs have rarely been considered as part of the network. Thus, data communication approaches have not even tried. The consequence is a suboptimal communication in terms of performance, usability and reliability. In this paper we present BCI as a paradigm in Wireless Body Area Networks (WBANs) that interfaces multisensorial sources with the user brain. The BCIWBAN concept treats cognitive processes as communication end-points in which sensorial information is consumed by cognitive processes. In the context of WBAN, BCIs implement the functionalities of the lower layers of communication open system: signal transduction into data, access control to the physical and cognitive media and data transmission. The BCI-WBAN paradigm extends the scope of WBAN from mere biosignal sensing by adding interpretation of neural correlates of cognitive information.
\end{abstract}

Keywords: Telemedicine, EEG, WBAN, Brain-computer Interface.

adfa, p. 1, 2011.

(C) Springer-Verlag Berlin Heidelberg 2011 


\section{Introduction}

The main purpose of BCI systems is to enable users to communicate with computers without muscles by interpreting and transmitting cognitive information from electrophysiological correlates. Along the last two decades, many definitions have been proposed for Brain-computer interfaces (BCIs), the most popular one, established that a BCI "is a communication system that does not depend on the brain's normal output pathways of peripheral nerves and muscles" [1]. Originally, it was intended for people with severe motor impairment (e. g. locked-in syndrome, Amyotrophic Lateral Sclerosis (ALS), etc.) with no other way of communication but the direct detection of the user volition from neurosignals. Currently, there are BCI works in diverse areas, such as cognitive rehabilitation, entertainment, education, neuromarketing, etc. [2]. The majority of BCIs are based on EEG signals because they can be sensed with noninvasive techniques and their price is relatively low even for small labs.

There are several types of BCI systems. For instance, BCIs based on Event-related potentials (ERPs) are ones of the most popular. ERPs are indicators of brain activities that occur in preparation for or in response to discrete external events [3]. Other BCIs use repetitive stimulus to evoke a permanent response (e.g. visual steady-state responses (SSVEP) [4], auditory steady-state response (ASSR) [5], etc.) whose energy can be modulated by cognitive task such as selective attention [6]. These potentials are narrow-bandwidth responses with high signal-to-noise ratio (SNR) that facilitates their processing and detection. Furthermore, there are BCIs based on the modulation of the amplitude of spectral bands. In this classification, we include those based on the amplitude modulation of sensory-motor rhythm by means of motor imagery [7], alpha band by means of mental switching from relaxation to arousal states, slow cortical potentials by means of own-user strategy, etc.

BCIs have improved usability and user comfort thanks to new advances in wireless technology and the use of new polymers and substrates for dry sensing (see [8] for a complete review). Now, the range of potential users is much larger, with applications in ambulatory EEG sensing for clinic testing, entertainment and other uses [9][10]. The most advanced BCI are low-cost, wearable and dry EEG headsets devices with wireless transmission that allows mobile EEG services [11][12]. However, and despite all these new features, both classical and current BCIs suffer from the same inconvenients.

Current and classical EEG-BCIs have some problems in common. First, EEG is a very noisy signal and any feature considered for the interpretation of the corresponding cognitive or mental state gives rise to poor inference. Typically, this problem has been overcome by grand-averaging trials (e.g. approximately one hundred for visual evoked potentials [13]). Assuming EEG to be stationary, the improvement in SNR follows equation (1) with $N$ the number of trials

$$
\text { SNRnew }(\mathrm{dB})=\text { SNRoriginal }(\mathrm{dB})+10 \log 10(\mathrm{~N})
$$


SNR improvement is the first step for throughput improvement. Throughput is other problem. Typically, BCI achieve an information transmission rate (ITR) of approximately between 0.3 to $0.6 \mathrm{bits} / \mathrm{s}$ [14]. Furthermore and as far as we know, the maximum reported ever, was 1.13 bits/s in 2003 [15]. Since then, and despite technologic advances, performance has not significantly improved. One of the reasons that could justify this disappointment is that other important aspects of BCI have not been changed. In this regards we could point out two aspects: data processing techniques and psycho-physiologic paradigms.

Apart of few exceptions, BCIs has not been treated as a technology suited for data networks. Brain signals are typically processed with intelligent algorithms of extraction, dimension reduction and classification of features that include complex multivariate analysis. In this regards we just cite some BCI studies based on Self-Organizing Map [16], Support Vector Machine theory [17], Artificial Neural Networks (ANNs) such as multilayer perceptron (MLP), etc. However, a simple analysis of the fundaments of BCIs would conclude that they really represent interfaces of a dta network. Then, typical data transmission issues such as media access and control, signal transduction, throughput improvement or data error correction deserve to be considered and applied to the brain as an entity of the data network. In summary, in BCI-WBANs a biological organ, our brain, is part of the network.

In this paper we present BCIs as a specific link of the data network that interface input sensorial information with cognitive processes in the context of WBAN. Taking this approach, conventional network procedures and assumption can be reused, thus giving rise to a much efficient interface.

The most important contribution in the BCI-WBAN paradigm is the definition of a cyber area network composed by electric and biologic entities in which data and information flows bidirectional and transparently

\section{The BCI-WBAN concept}

\subsection{Definition and overall concept}

\section{BCI definition}

In BCI literature there are many definitions of BCI. For instance, the first one was given in 1973 by Vidal who stated "The BCI system is geared to use both the spontaneous EEG and the specific evoked responses triggered by time-dependent stimulation under various conditions for the purpose of controlling such external apparatus as e.g. prosthetic devices" [18]. Another definition giving in 2002 by expert established that a direct brain-computer interface is a device that provides the brain with a new, non-muscular communication and control channel [1]. However, the most popular definition was given in 2000 by a group of prestigious neuroscientifics during the First International Meeting of BCIs [19].

\section{BCI-WBAN definition}


We define BCI-WBAN as the networking area that encompasses from sources of multi-sensorial information to our brain. Multisensorial sources are multimedia devices, speakers, TV, audio-head set, traffic lights, etc. Other devices related to clinic and rehabilitation are also considered (e. g. electric, tactile, haptic, visual, auditory or somatosensory stimulators). There are also biological sources. For instance, voices in multi-taker scenarios (e.g. cocktail party phenomenon [20]), human faces, etc. Conversely, in the brain side we consider cognitive processes that perceive, attend, interpret these sensorial signals and finally take a decision. Decisions can be taken either directly based on these sensorial signals or not.

Then, BCIs constitute the communication interface in the WBAN that, based on brain signals, extract relevant cognitive information generated by brain structures and generates and output stream of data. Typically, this data stream is used to feedback the sensorial source, thus closing the communication loop. Also, it can be used for communication of cognitive states (e.g. level of attention, tiredness, arousal), interest or endogenous context update as response to a novel and relevant sensorial information. Some examples of application of BCI-WBAN [21] could be the online measure of cognitive load in dangerous works, a communication speller for people suffering from locked-in syndrome or ALS [22], or as an attention/interest detector with potential application in neuromarketing or Ambient Assisted Living (AAL).

\subsection{Architecture}

\section{BCI}

BCI architecture was firstly defined by [23]. A typical implementation is the EEGBCI, which is compounded of the following blocks (see Fig. 1):

- EEG Acquisition System: electrodes, front-end amplifier, A/D converter

- Signal processing: Signal pre-processing, feature extraction and classification.

- Application Interface: This block provides feedback to the BCI user and also interfaces devices, sensors and actuators

- User interface: Any communication system needs mechanism to feeback emisor with the state of the communication. In BCIs, a display is typically used to present the BCI user the results. Then BCI users can on-line modify their cognitive actions to obtain the optimal result. 


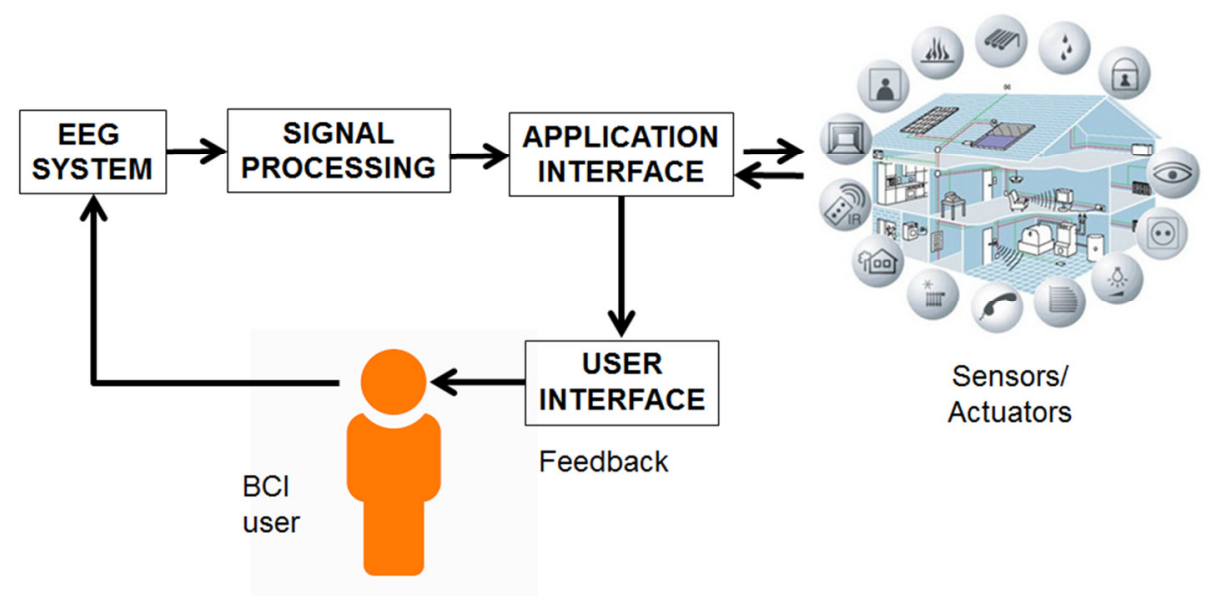

Fig. 1. BCI architecture. Adapted from [23].

\section{BCI-WBAN}

BCI-WBAN architecture is compounded of the blocks and elements needed to present multisensorial information to user and transmit out cognitive information. Figure 2 illustrates a layered version of the BCI-WBAN architecture. Firstly, a source transmits sensorial signals to the user. User receives the signals by means of receptors or sense organs. Receptors transduce this sensorial information into electrical currents that reach cortical regions in our brain via afferent pathway.

- If the context of the OSI model these processes occur in the physical layer. In the context of BCI-WBAN ,these processes occur in the physiological layer.

These electro-physiological signals reach cognitive structures in the cognitive media and trigger concomitant cognitive processes, such as perception, attention, cognition and volition. Typically, these processes temporally correlate with middle-latency potentials (approx. $100 \mathrm{~ms}$. after stimulus onset). In our daily routine, we are rarely surrounded by just a sensorial source. Conversely, we are always immersed in a mess of multi-sensorial stimuli that fight to grab our attention. For instance, during our daily commuting we listen the radio, hear traffic noise, see traffic lights, hear policeman shouting and our navigator given instructions at the same time. Although attention in more than one stimulus is possible [24], human beings are tailored to focus our attention in one relevant stimulus and filter out the rest. The latter is the so-called selective attention. Attention, in its several flavors (selective, pre-attentional, dichotic, etc.) plays the role of referee in the access to cognitive media where cognitive processes occur. Then, attention implements the media-access layer.

- If the context of the OSI model, control to the access media ocurr in the link layer. In the context of BCI-WBAN, the access to the cognitive media is executed by the cognitive task called attention. 
Once user have perceived, recognized and interpreted the external information, then decides and action. A simple action that user could take could be switch attention to another stimulus. When that happens, top-down mechanisms occurs that cause modulation of the energy of signals in physical layer by enhancing of that of the target stimuli and attenuating that of the distracters. In turns, the latter causes and diminishing of the energy in the bottom-up direction toward upper cognitive layers. In turns, that facilitates the attentional effort needed as media-access controller of the incoming stimuli. The latter constitutes a loop (see Fig. 2).

- If the context of the OSI model, execution of actions as a consequence of volition is introduced by users via the application layer. In the context of BCI-WBAN, the access to the cognitive media is executed by executive system of our brain.

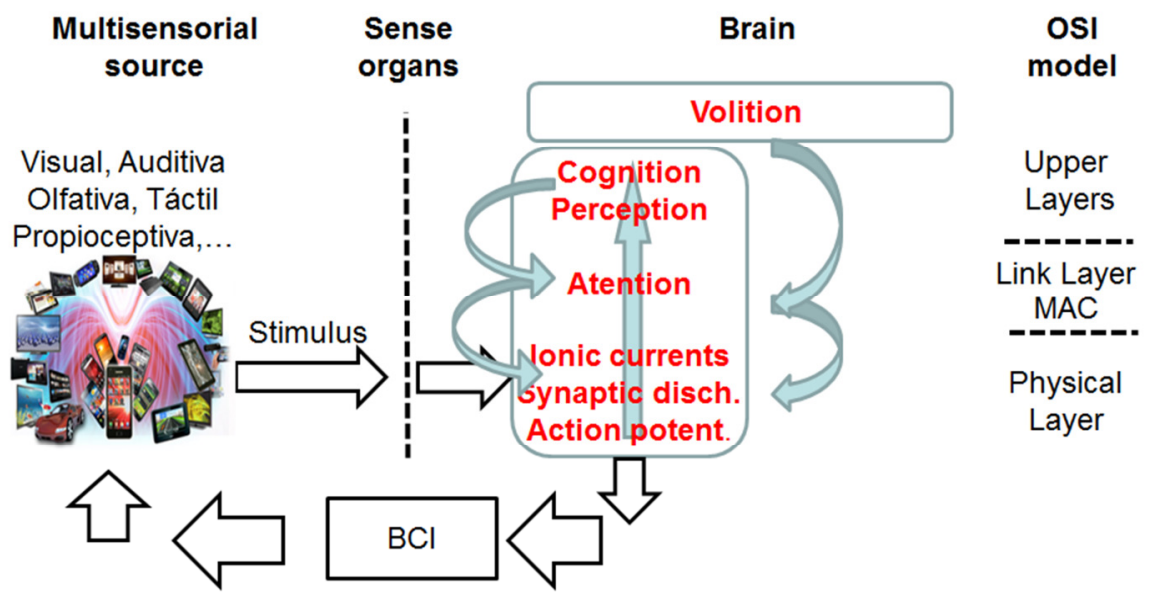

Fig. 2. Layered version of the BCI-WBAN architecture. On the left, external devices present $\mathrm{BCI}$ user sensorial stimuli. In the center, sense organs catch them and transform their mechanical energy into electrical currents. Electrical currents trigger cognitive processes in a bottomtop directon. Executive functions cause modulation of the electrical signal in top-bottom direction. BCI measure this top-down influence and feedback the external source (or other network enteities). On the right, the equivalence between the BCI-WAN layered model and OSI model.

\subsection{BCI-WBAN in the network}

Figure 3 shows full application model that includes not only the elements of BCIWBAN described in previous sections, but elements of remote connections in the context of Wide Arena Networks (WANs). This general model would enable teleservices based on on-line cognitive rehabilitation. Currentely there studies of telematics services for remote cognitive rehabilitation. However, these proposal lack of interactivity [25]. The BCI-WBAN architecture complements these services by offering 
the user mobility, usability and on-line feedback during the execution of the program. The components and elements of the BCI-WBAN concept of the proposed teleservice are:

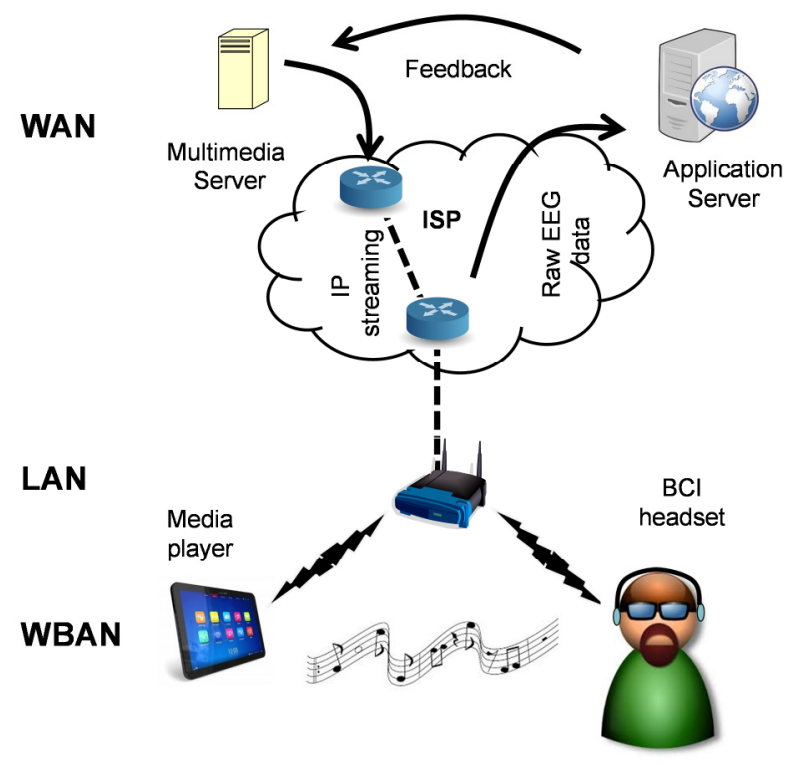

Fig. 3. Application model of BCI-WAN for a remote service.

\section{WAN.}

- Application server: The main application runs in this server. It is responsible for:

- User control interface: It offers a GUI to person in charge of the execution of the session (e. g. in the context of application such as remote EEG testing for clinical purposes, rehabilitation programs, etc.)

- Sensorial stimulation control: It triggers sensorial programs stored in a multimedia server that, in turns, streams down them to the media player.

- Signal processing: This function inputs EEG signals form EEG headset and process them to obtain an output data stream.

- Multimedia server: It stores a collection of multimedia programs that users could use.

\section{LAN}

- Access point: Typically, a wireless router.

\section{WBAN}

- Media player: It plays the multimedia stimulus streamed from Multimedia server. 
- BCI headset: It acquires EEG signals and transmit them toward the Application server.

\section{$3 \quad$ Related works}

As stated in the introduction section there are few studies in which BCIs are considered as communication interfaces of WBANs or BANs. In these studies, communication techniques such as analog and digital modulations and constellation of signals are used to detect and transmit out information generated in the cognitive media. In this section we mention some pioneers works that took benefits of the BCI principles to achieve an efficient performance. At the time of some of these studies, BCI were wired devices. Then, BCI-WBANs should be interpreted in the context of BANs.

We present two categories, one for visual and another for auditory sensorial information. In both cases, we present studies that combined hybrid digital communication techniques with priors about the modulating effect of cognitive task on brain signals to detect and transmit out cognitive information.

\section{Visual BCIs.}

The BCI that achieved the highest throughout was developed by Gao in 2003 and achieved a throughput of $1.13 \mathrm{bits} / \mathrm{s}$ [15]. The extraordinary performance in this study was based upon the supposition that user were able to gaze at different stimulus. However, BCI users with the ability to gaze would likely opt for a much faster and simpler technology such as eye-trackers. Bearing in mind this limitation, authors in [26] developed a high performance BCI without the need of gazing. At that moment, this was an extraordinary advantage in comparison with the rest of visual BCIs that requested gazing. Despite this handicap, the maximum throughout achieved was $0.64 \pm 0.27 \mathrm{bits} / \mathrm{s}$ with peaks of $1.16 \mathrm{bits} / \mathrm{s}$ and accuracy (or 1-BER) of $90 \pm 7 \%$, which is an excellent performance for a binary BCI. The most important factor that contributed to this excellent performance was the use of customized visual stimulation whose corresponding brain response constituted a constellation of two signals. Then, detection was performed with a coherent on-off keying receiver (OOK) based on a bank of two correlators (see Fig 4.1).

With the idea use digital receiver to detect symbols in a constellation of brain signals described in the previous paragraph, in [27] and [28] the number of symbols in the EEG constellation increased by reusing the same frequency (see Fig. 4.2). This was a novel approach in visual BCIs. Till that moment, phase was not been widely used in on-line detection. Instead, most of the visual BCI just asynchronously measure energy of brain activity. The reason for that is the difficulty to keep accurate synchrony between the stimulation unit and the EEG acquisition system. The advantage introduced in these two studies was the use of a coherent detection, in which information of phase is used to detect the correct symbol from the constellation. According to theory, the gain introduced by a coherent OOK receiver is approximately $3 \mathrm{dBs}$ in comparison with the non-coherent. Then, this efficient design of BCI attained better performance. 

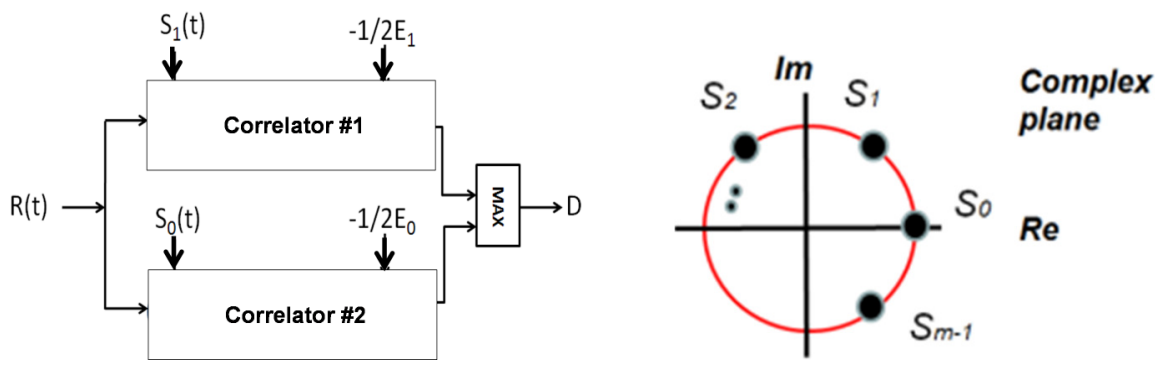

Fig. 4. (1) Bank of correlators that implement a coherent OOK receiver with decisions based on maximum a posteriori criterion. The signal in reception $(R(t))$, passed through two correlators that also input a replica of the signals of the constellation $\left(S_{I}(t)\right.$ and $\left.S_{2}(t)\right)$ and the energy of each one $\left(E_{1}\right.$ and $\left.E_{2}\right)$; (2) Constellation of m signals of a PSK modulation. Adapted from [27] and [28].

\section{Auditory BCIs.}

Originally, BCIS were meant for people unable to do muscular movements. However, visual BCIs contradict the original definition of BCIs because they require the subjects to gaze, explore and coordinate the eyes, thus causing muscular movement [29]. One important reason of the existence of auditory BCIs is that they do not require the user to gaze, thus being suitable even for people with visual impairment.

In [30], a tailored auditory stimulation was design to evoke auditory steady-state responses (ASSR). ASSRs are composed of a train of superimposed auditory brainstem responses, that added in phase, conforms an averaged response with most of the energy located around the frequency of repetition [6]. In this case, detection of cognitive information was performed by building a constellation of two signals. The authors of this study took advantage of well-known properties of the physiology of the human auditory system that, when stimulated with an AM modulated signal, can be described as a non-coherent AM demodulator. Figure 5 illustrates the following example: when we present a stimulus consisting of a carrier (e.g. $1 \mathrm{Khz}$ ) AM modulated by a sinusoidal wave (e.g. $100 \mathrm{~Hz}$ ) the transduction from mechanical energy into electrical nerve impulses is performed in such a way that only semi-cycles of the same sign (e. g. positive) of the incoming wave evoke electrical nerve impulses. The overall effect is a rectification of the shape of the sound that, after low-band filtered, works as envelope detector. Then, the modulating signal of $100 \mathrm{~Hz}$, is recovered from EEG as if it were an AM receptor. This principle, which is broadly used in clinical testing for assessment of hearing impairment [31] was first used in this study to detect and transmit out information from the cognitive media. 


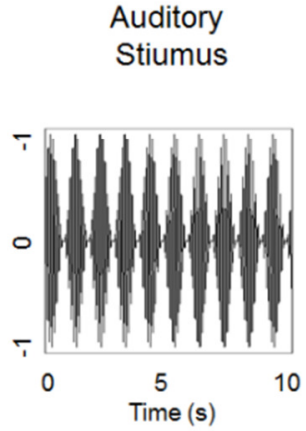

Rectification

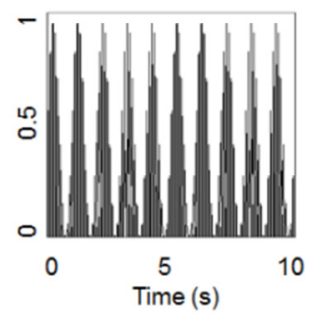

EEG Power Spectral Density

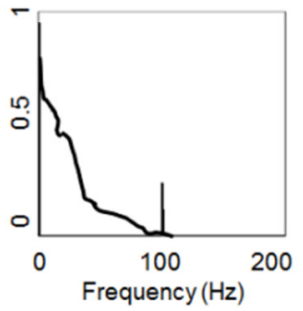

Fig. 5. Rectification of an AM modulated stimulus by human auditory system. The first plot shows one second window of a sinusoidal wave of frequency $1 \mathrm{Khz}$ (carrier) AM modulated by a pure tone of $100 \mathrm{~Hz}$. The plot in de middle shows the rectification process caused by hair cells in the cochlea and other neural structures of the human auditory system. The plot on the right shows the Power Spectral Density of a typical EEG acquisition in which the modulating pure tone of $100 \mathrm{~Hz}$ appears with high SNR. In summary, behavior of the human auditory system when stimulated with a AM modulated signal, corresponds to that of a non-coherent AM receiver.

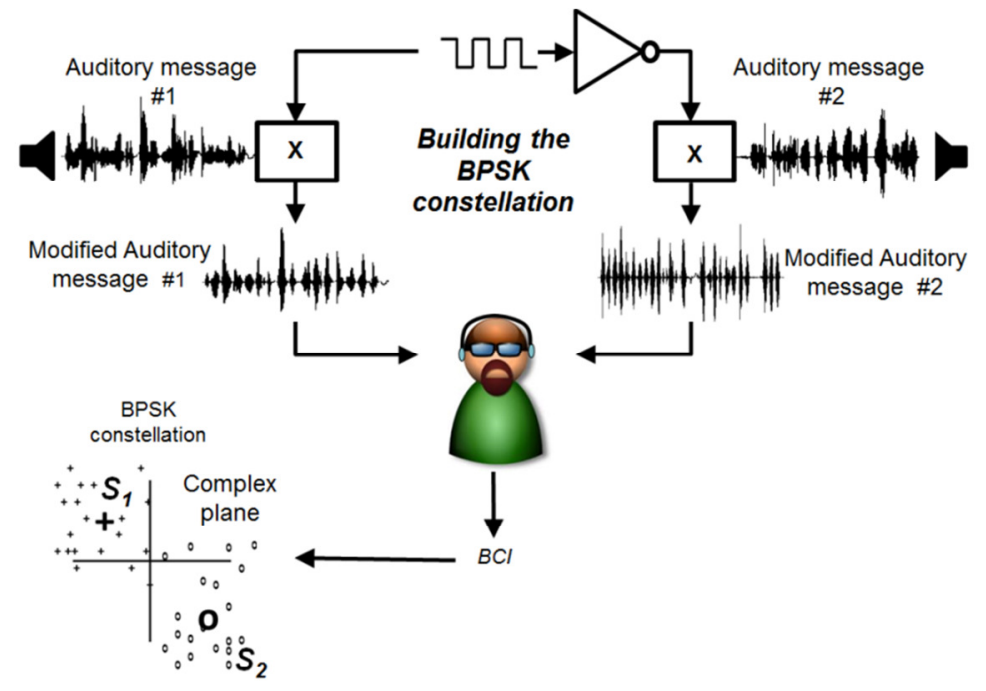

Fig. 6. BPSK system for detection of attention. This schema is part of a dichotic listening task, in which user must pay attention to one stimulus and ignore the other. To facilitate detection of the attended stimulus, both stimulus were perturbed by modulating their envelope by two counter-phases pure tone of the same frequency. The effect of the perturbation was the generation of a BPSK constellation on brain signals. Adapted from [32]. 
The most clear example in BCI literature of the use of the BCI as a digital communication system in the context of BANs was developed in [32]. The authors of this study presented a procedure based on the binary phase-shift keying (BPSK) receiver that permitted detection of selective attention to human speeches in real-time. They used barely-audible counter-phased perturbations of the speeches in such a way that the joined EEG response (speeches-perturbations) elicited a robust BPSK constellation (see Fig. 6). Then attention was detected and transmitted by mean of BPSK receiver. The results confirmed the expected generation of the BPSK constellation by the human auditory system. Furthermore, evaluation of performance in terms of throughput and BER was measured under different conditions of SNR and the results fairly matched the theoretical. The latter was an extraordinary advance in the context of BCI as communication system in BANs. Other studies such as [33][34] tried similar approaches with similar success.

\section{The PLAT-EEG project.}

The BCI-WBAN is a concept still to develop. Despite some pioneers studies mentioned in previous section, more steps must be takenamong BCI researches. In this section we briefly present a project that, based on the principles of BCI-WBAN, moves forward this direction.

PLAT-EEG aims for the development of a high performance platform for the acquisition, processing of neurosignals, detection and remote transmission of cognitive information (see Fig. 7). The platform will support common services and resources to offer tele-services and BCI applications, for instance, on-line sensing of neurosignals, or cognitive programs for rehabilitation. Among potential users of this platform, we mention people with sever motor and muscular impairment, people suffering from attention disorders, etc.

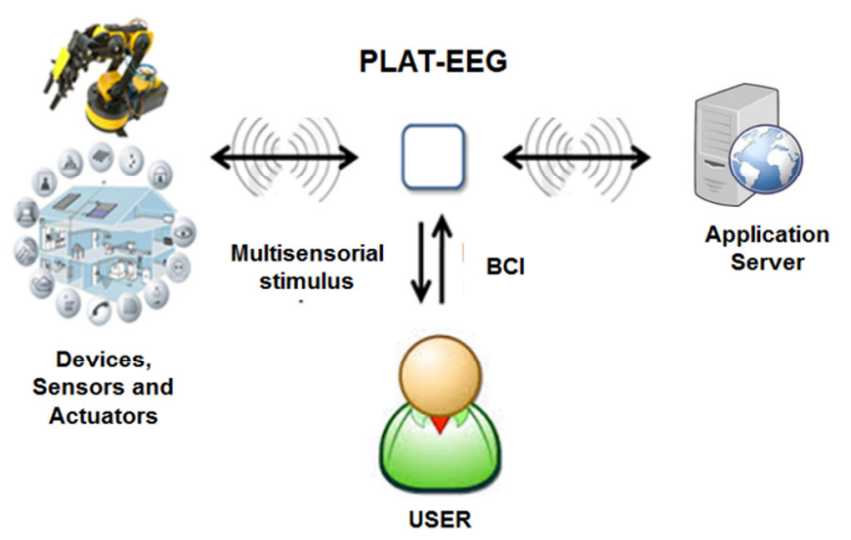

Fig. 7. Basic architecture of PLAT-EEG Adapted from [35]. 


\section{Conclusions}

Traditionally, BCIs have not been considered as part of WBANs. The consequence is a suboptimal communication interface in terms of performance. In this paper we have introduced the BCI-BAN concept as a novel paradigm in data communication between biological and electronic entities that encompass the area network between multimedia sources and the user brain. In the context of BCI-WBAN, cognitive processes are communicating entities that inputs sensorial information originated by multimedia devices and output cognitive information by means of a BCI. In the context of WBAN, BCIs implement the functionality of the lower layers of an interconnection open system, namely physical and access control to the cognitive media, thus providing output transmission functionality to cognitive structures. BCIs extends the scope of Personal or Body Area Networks by including the brain in the last mile of the communication network.

\section{Acknowledgement}

This study has been co-financed by Nicolo Association for the R+D+i in Neurotechnologies for disability, the regional project P11-TIC-7983, Junta of Andalucia (Spain), the National Grant TIN2012-32030 (Spain), co-financed by the European Regional Development Fund (ERDF) and the CASIP research group TIC-117.

\section{References}

1. J. R. Wolpaw, N. Birbaumer, D. J. McFarland, G. Pfurtscheller, and T. M. Vaughan, "Braincomputer interfaces for communication and control," Clin. Neurophysiol., vol. 113, pp. 76791, 2002.

2. R. Ohme, D. Reykowska, D. Wiener, and A. Choromanska, "Application of frontal EEG asymmetry to advertising research," Journal of Economic Psychology, vol. 31, no. 5, pp. 785-793, Oct. 2010.

3. J. Cacioppo, L. Tassinary, G. Berntson (Eds), Handbook or Psychophysiology, 2nd Edition. Cambridge University Press 2000.

4. M. Middendorf, G. McMillan, G. Calhoun, and K. S. Jones, "Brain-computer interfaces based on the steady-state visual-evoked response," IEEE Transactions on Rehabilitation Engineering, vol. 8, no. 2, pp. 211-214, Jun. 2000.

5. Do-Won Kim, Jae-Hyun Cho, Han-Jeong Hwang, Jeong-Hwan Lim, and Chang-Hwan Im, "A vision-free brain-computer interface (BCI) paradigm based on auditory selective attention," 2011, pp. 3684-3687.

6. S. A. Hillyard, R. F. Hink, V. L. Schwent, and T. W. Picton, "Electrical Signs of Selective Attention in the Human Brain," Science, vol. 182, no. 4108, pp. 177-180, Oct. 1973.

7. G. Pfurtscheller, C. Neuper, Motor imagery activates primary sensorimotor area in humans, Neuroscience Letters 239 (1997) 65-68.

8. M. Lopez-Gordo, D. Morillo, and F. Pelayo, "Dry EEG Electrodes," Sensors, vol. 14, no. 7, pp. 12847-12870, Jul. 2014. 
9. A. Nijholt, D. P.-O. Bos, y B. Reuderink, «Turning shortcomings into challenges: Braincomputer inter-faces for games», Entertain. Comput., vol. 1, n.o 2, pp. 85-94, abr. 2009.

10. P. Chang, K. S. Hashemi, y M. C. Walker, «A novel telemetry system for recording EEG in small ani-mals», J. Neurosci. Methods, vol. 201, n.o 1, pp. 106-115, sep. 2011.

11. S. Lee, Y. Shin, S. Woo, K. Kim, y H.-N. Lee, «Review of Wireless Brain-Computer Interface Systems», en Brain-Computer Interface Systems - Recent Progress and Future Prospects, R. Fazel-Rezai, Ed. InTech, 2013.

12. N. S. Dias, J. P. Carmo, P. M. Mendes, and J. H. Correia, "Wireless instrumentation system based on dry electrodes for acquiring EEG signals," Medical Engineering \& Physics, vol. 34, no. 7, pp. 972-981, Sep. 2012.

13. J. V. Odom, M. Bach, M. Brigell, G. E. Holder, D. L. McCulloch, A. P. Tormene, and Vaegan, "ISCEV standard for clinical visual evoked potentials (2009 update)," Documenta Ophthalmologica, vol. 120, no. 1, pp. 111-119, Feb. 2010.

14. M. Cheng, X. Gao, S. Gao, Senior Member, IEEE, and D. Xu, "Design and Implementation of a Brain-Computer Interface With High Transfer Rates", IEEE Transactions on Biomedical Engineering, vol. 49, no. 10, October 2002, pp. 1181-1186.

15. X. Gao, D. Xu, M. Cheng, and S. Gao, "A BCI-Based Environmental Controller for the Motion-Disabled," IEEE Transactions on Neural Systems and Rehabilitation Engineering, vol. 11, pp. 137-140, Jun. 2003.

16. H. Liu, J. Wang, C. Zheng, "Using Self-organizing Map for Mental Tasks Classification in Brain-Computer Interface", Lecture Notes in Computer Science, Vol. 3497 (Springer, Berlin, 2005) pp. 327-332.

17. X. Liao, D. Yao, C. Li, "Transductive SVM for reducing the training effort in BCI.", J. Neural Eng. 4 (3) (2007) pp. 246-254.

18. J. J. Vidal, "Toward Direct Brain-Computer communication," Annu. Rev. Biophys. Bioeng., pp. 157-180, 1973.

19. J. R. Wolpaw, N. Birbaumer, W. J. Heetderks, D. J. McFarland, P. H. Peckham, G. Schalk, E. Donchin, L. A. Quatrano, C. J. Robinson, and T. M. Vaughan, "Brain-Computer Interface Technology: A Review of the First International Meeting," IEEE Transactions on Rehabilitation Engineering, vol. 8, no. 2, pp. 164-173, Jun. 2000.

20. E. C. Cherry, "Some Experiments on the Recognition of Speech, with One and with Two Ears," The Journal of the Acoustical Society of America, vol. 25, no. 5, pp. 975-979, 1953.

21. B. Blankertz, M. Tangermann, C. Vidaurre, S. Fazli, C. Sannelli, S. Haufe, C. Maeder, L. Ramsey, I. Sturm, G. Curio, and K.-R. Müller, "The Berlin Brain-Computer Interface: NonMedical Uses of BCI Technology," Front. Neurosci., vol. 4, 2010.

22. N. Birbaumer, N. Ghanayim, T. Hinterberger, I. Iversen, B. Kotchoubey, A. Kübler, J. Perelmouter, E. Taub, and H. Flor, “A spelling device for the paralysed," Nature, vol. 398, no. 6725, pp. 297-298, Mar. 1999.

23. M. Moore, "Real-World Applications for Brain-Computer Interface Technology", IEEE Transactions on Neural Systems and Rehabilitation Engineering, vol. 2, no. 2, June 2003.

24. [M. M. Müller, P. Malinowski, T. Gruber, and S. A. Hillyard, "Sustained division of the attentional spotlight," Nature, vol. 424, no. 6946, pp. 309-312, Jul. 2003.

25. García Vázquez, Carolina; Moreno Martínez, Esther; Valero Duboy, Miguel Angel; Martínez Juez, María Teresa y Torre Calero, Mari Satur, "Servicio ubicuo de estimulación cognitiva orientado a personas con enfermedad de Parkinson". In "XI Jornadas de Ingeniería Telemática (JITEL 2013). Granada, October 2013. 2013

26. M. A. Lopez-Gordo, F. Pelayo, and A. Prieto, "A high performance SSVEP-BCI without gazing," in The 2010 International Joint Conference on Neural Networks (IJCNN), Barcelona, Spain, 2010, pp. 193-197. 
27. M. A. Lopez-Gordo, A. Prieto, F. Pelayo, and C. Morillas, "Use of Phase in BrainComputer Interfaces based on Steady-State Visual Evoked Potentials," Neural Process Lett, vol. 32, no. 1, pp. 1-9, May 2010.

28. M.-A. Lopez, H. Pomares, A. Prieto, and F. Pelayo, "Multiple AM Modulated Visual Stimuli in Brain-Computer Interface," in Bio-Inspired Systems: Computational and Ambient Intelligence, vol. 5517, J. Cabestany, F. Sandoval, A. Prieto, and J. M. Corchado, Eds. Berlin, Heidelberg: Springer Berlin Heidelberg, 2009, pp. 683-689.

29. M. A. Lopez-Gordo, R. Ron-Angevin, and F. Pelayo Valle, "Auditory Brain-Computer Interfaces for Complete Locked-In Patients," in Advances in Computational Intelligence, vol. 6691, J. Cabestany, I. Rojas, and G. Joya, Eds. Berlin, Heidelberg: Springer Berlin Heidelberg, 2011, pp. 378-385.

30. M. A. Lopez, H. Pomares, M. Damas, A. Prieto, and E. M. Plaza Hernandez, "Use of Kohonen Maps as Feature Selector for Selective Attention Brain-Computer Interfaces," in Bioinspired Modeling of Cognitive Tasks, vol. 4527, J. Mira and J. R. Álvarez, Eds. Berlin, Heidelberg: Springer Berlin Heidelberg, pp. 407-415.

31. [A. J. Moreno-Aguirre, E. Santiago-Rodríguez, T. Harmony, A. Fernández-Bouzas, and E. Porras-Kattz, "Analysis of auditory function using brainstem auditory evoked potentials and auditory steady state responses in infants with perinatal brain injury," International Journal of Audiology, vol. 49, no. 2, pp. 110-115, Feb. 2010.

32. M. A. Lopez-Gordo and F. Pelayo, "A Binary Phase-Shift Keying Receiver for the Detection of Attention to Human Speech," International Journal of Neural Systems, p. 130418190845004, Mar. 2013.

33. M. A. Lopez-Gordo, F. Pelayo, A. Prieto, and E. Fernandez, "An Auditory Brain-Computer Interface with Accuracy Prediction," International Journal of Neural Systems, vol. 22, no. 3, pp. 1-14, 2012.

34. M. A. Lopez-Gordo, E. Fernandez, S. Romero, F. Pelayo, and A. Prieto, "An auditory brain-computer interface evoked by natural speech," Journal of Neural Engineering, vol. 9, no. 3, pp. 1-9, Jun. 2012.

35. M. A. Lopez-Gordo, $M^{a}$ Ángeles Martín Sánchez, E. M. de la Plaza Hernández, F. Pelayo Valle, "Plataforma de Altas Prestaciones para la Adquisición, Extracción y Procesamiento Inteligente de Señales EEEG y Telemonitorización (PLAT-EEG)", Cognitive Area Networks, vol. 1, $\mathrm{n}^{\circ}$ 1, Junio 2014, Asociación Nicolo, ISSN: 2341-4243 Actas del $6^{\circ}$ Simposio CEA Bioingeniería 2014, Interfaces Mente-computador y Neurotecnologías, Granada Junio 2014. 